\title{
Chiral Ag Nanostructure Arrays as Optical Antennas
}

\author{
J. J. Gough ${ }^{1}$, D. McCloskey ${ }^{1}$, J. M. Caridad ${ }^{1,2}$, V. Krstic ${ }^{1,3}$, M. Müller ${ }^{4}$, N. Gaponik ${ }^{4}$ and A. L. Bradley $^{1}$ \\ ${ }^{1}$ Trinity College Dublin, Photonics, School of Physics and CRANN, Dublin 2, Ireland \\ 2 Technical University of Denmark, Department of Micro- and Nanotechnology, Lyngby, Denmark. \\ ${ }^{3}$ FAU Erlangen-Nürnberg, CEEAM, Nägelsbachstrasse, Erlangen, Germany. \\ ${ }^{4}$ TU Dresden, Physikalische Chemie, Helmholtzstraße, Dresden, Germany \\ goughj3@tcd.ie
}

\begin{abstract}
Directional and circularly polarised emission from quantum dots coupled with chiral Ag nanostructures has been demonstrated. Semiconductor quantum dots were deposited onto the chiral structures using the Layer-by-Layer deposition technique. Time-resolved photoluminescence measurements demonstrated a lifetime quenching efficiency of $82 \%$, confirming the strong interaction between the emitters and the antennas. Angle- and polarisation-resolved photoluminescence measurements reveal that the chiral nanostructures influence the far-field emission properties of the quantum emitters. The far-field emission from the quantum dots coupled to the chiral nanostructures displays directionality in the emission pattern and more than $17 \%$ circular polarisation, following the handedness of the chiral structure.
\end{abstract}

\section{INTRODUCTION}

Plasmonic metal nanostructures exhibiting chiroptical effects in the visible spectrum, either intrinsically or via coupling to chiral molecules, have been the subject of intensive theoretical investigation in recent years [1]. Recent advances in lithographic [2]-[3] and molecular self-assembly [4] techniques have paved the way for the fabrication of a new generation of three dimensional anisotropic structures having chiroptical responses at optical frequencies [2]-[4]. While these chiroptical properties are of significant importance for this work, an equally important topic is that of optical antennas [5]. Plasmonic nanostructures can be used to concentrate, control and direct electromagnetic radiation at optical frequencies [6]. Chiral antennas with helical geometries have the added ability to polarise the emitted radiation according to the handedness of the helix [7]. Herein, we demonstrate directional and circularly polarised emission from semiconductor quantum dots (QDs) coupled to chiral Ag nano-antennas.

\section{EXPERIMENTAL}

Arrays of right handed chiral Ag nanostructures were fabricated on an ITO substrate using a room temperature glancing angle vapour deposition (GLAD) technique [5]. The nanostructures approximate to a helical shape with a pitch of $90 \mathrm{~nm}$, diameter of $80 \mathrm{~nm}$ and a height of $210 \mathrm{~nm}$. Negatively charged thiolglycolic acid (TGA) stabilised Cadmium Telluride (CdTe) QDs with a diameter of $2.6 \mathrm{~nm}$ in the aqueous solution were used for Layer-by-Layer (LBL) deposition. With the LBL technique, alternate polyelectrolyte and nanoparticle monolayers are deposited via electrostatic attraction. This technique allows precise control over the separation between each monolayer of QDs and the distance between the Ag nanostructures and the initial layer of QDs. The polyelectrolytes used were positively charged poly[diallyl dimethyl ammonium chloride] (PDDA) and negatively charged poly[sodium 4-styrenesulphonate] (PSS). Both polyelectrolyte solutions were prepared to a concentration of $0.1 \mathrm{M}$. Two bilayers of PDDA/PSS and a final layer of PDDA were deposited onto the Ag nanostructures before depositing the QDs. Each bilayer has a thickness of $\sim 3 \mathrm{~nm}$ and therefore the separation between the Ag nanostructures and the initial layer of QDs is $\sim 7.5 \mathrm{~nm}$. To increase the emission intensity from the QDs a total of 5 layers of QDs were deposited, with each layer of QDs separated by a PDDA layer. The final distance between the $\mathrm{Ag}$ nanostructures and the outermost layer of QDs was $\sim 25 \mathrm{~nm}$.

\section{RESULTS}

The chiral Ag nanostructure arrays were optically characterised by means of circularly polarised transmission 
and reflection measurements. The circular dichroism (CD) spectrum was obtained from the extinction spectra for left circularly and right circularly polarised light (LCP and RCP, respectively).

(a)

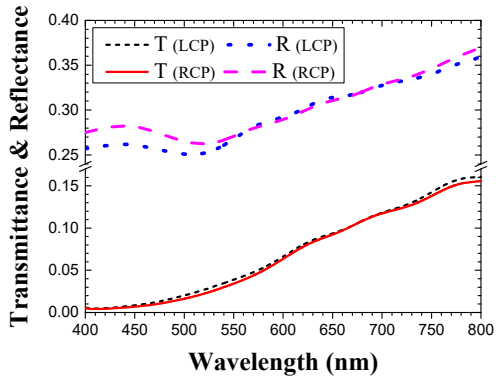

(b)

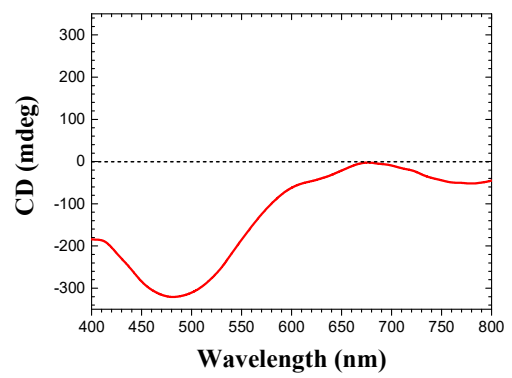

Fig. 1. (a) Transmittance and reflectance of a right handed chiral Ag nanostructure array for both circular polarisations, and (b) CD spectrum for the same array.

The circularly polarised transmission and reflection spectra in Fig. 1(a) demonstrate that the array of chiral Ag nanostructures absorbs and reflects more light that is polarised in the same handedness as the structure itself. The CD spectrum in Fig. 1(b) clearly demonstrates a strong circular dichroism with a peak value of -320 mdeg at 480 $\mathrm{nm}$. Photoluminescence (PL) and time-resolved PL (TRPL) measurements were performed after the deposition of the QDs to monitor the fluorescence intensity and decay dynamics.
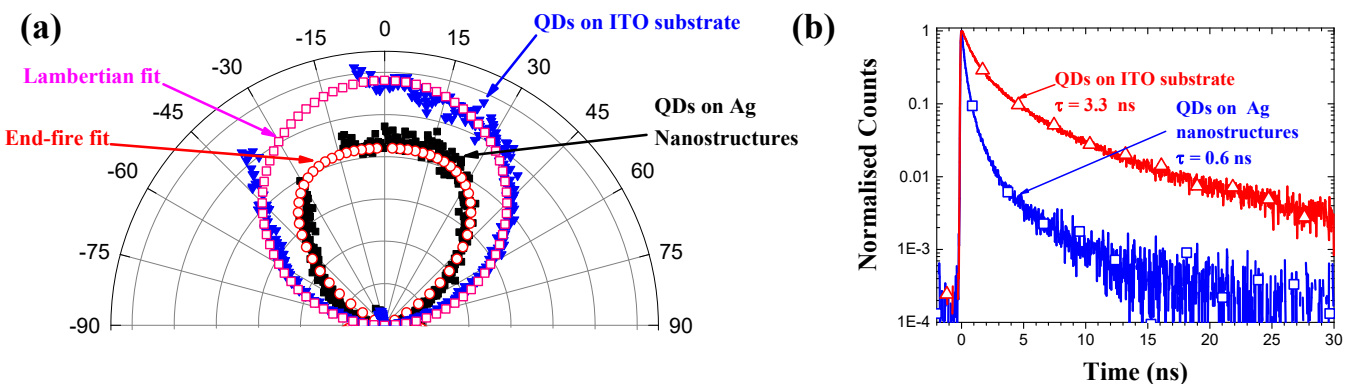

Fig. 2. (a) Measured emission pattern from the QDs coupled to the array of Ag nanostructures (solid square) with an ordinary end-fire fit (open circle), and measured emission pattern from the QDs on a bare ITO substrate (solid triangle) with a Lambertian fit (open square), and (b) QD fluorescence decay curves for QDs coupled to the Ag nanostructures (open square) and on the bare ITO substrate (open triangle).

The far-field emission patterns from the QDs coupled to the array of Ag nanostructures and the QDs on bare ITO can be seen in Fig. 2(a). The emission pattern from the QDs on the nanostructures (solid square) shows higher directionality than that of the QDs on the bare ITO substrate (solid triangle). The emission pattern from the QDs on the bare ITO follows that of a Lambertian $(\cos (\theta))$ dependence (open square) whereas the emission pattern from QDs coupled to the chiral Ag nanostructures fits with that of an ordinary end-fire pattern (open circle). The QDs coupled to the Ag nanostructures display $62 \%$ of the emitted power in the $30^{\circ}$ cone normal to the array plane as opposed to $50 \%$ of the power in the same cone for the QDs on the bare ITO substrate, as calculated from the fit for each pattern. The photoluminescence decay curves for the QDs coupled to the Ag nanostructures and on a bare ITO substrate are shown in Fig. 2(b). The QDs on the bare ITO (open triangle) have a lifetime of $3.3 \pm 0.1 \mathrm{~ns}$ compared to the QD lifetime of $0.6 \pm 0.1 \mathrm{~ns}$ for the QDs coupled to the nanostructures (open square). This yields a quenching efficiency, $\eta_{q}$ of $82 \pm 1 \%$, where the quenching efficiency is given by;

$$
\eta_{q}=1-\left(\tau_{Q D-A g} / \tau_{Q D-I T O}\right)
$$


Polarisation-resolved emission measurements were carried out using a LCP, continuous wave, $405 \mathrm{~nm}$ laser with an output power of $2.5 \mathrm{~mW}$. The polarisation-resolved emission components for LCP (dash) and RCP collection (solid) are shown in Fig. 3(a).

(a)

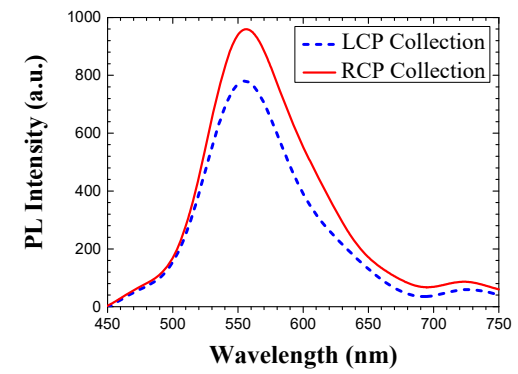

(b)

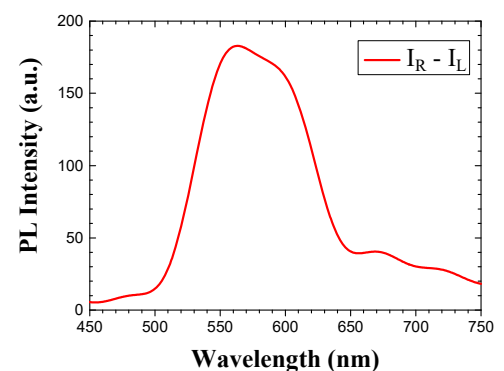

Fig. 3. (a) LCP (dash) and RCP (solid) emission components measured under LCP excitation, and (b) the difference between the emission components.

The emission spectra in Fig. 3(a) shows a higher intensity of RCP emission, which is in accordance with the handedness of the structure. The excess emission $\left(I_{R}-I_{L}\right)$ is shown in Fig. 3(b). The percentage of circularly polarised emission, $\rho_{c}$ is given by

$$
\rho_{c}=I_{R}-I_{L} / I_{R}+I_{L}
$$

Approximately, $17 \%$ of the total emission is right circularly polarised, demonstrating that the chiral nanostructures can impart their antenna properties to the nearby emitters.

\section{CONCLUSION}

The presence of preferentially circularly polarised emission indicates that the overall symmetry of the system has a notable influence on the emission of a quantum emitter. The circularly polarised photons are polarised in accordance with the handedness of the structure. Furthermore, as a result of the coupling between the antenna array and QD emitters, the emission from the QDs has higher directionality than for QDs on a bare substrate.

\section{ACKNOWLEDGEMENT}

This work was supported by the Irish Research Council, Grant No. (GOIPG/2013/680).

\section{REFERENCES}

[1] Fan, Z., Govorov, A. O., "Helical metal nanoparticle assemblies with defects: plasmonic chirality and circular dichroism". J. Phys. Chem. C, 115, 13254-13261, 2011.

[2] Mark, A. G., Gibbs, J. G., Lee, T.-C., Fischer, P. "Hybrid nanocolloids with programmed three-dimensional shape and material composition". Nature Materials, 12, 802-807, 2013.

[3] Caridad, J. M., McCloskey, D., Donegan, J. F., Krstic. V. "Controllable growth of metallic nano-helices at room temperature conditions”. Applied Phys. Lett, 105, 233114-1, 2014.

[4] Kuzyk, A., Schreiber, R., Fan, Z., Pardatscher, G., Roller, E.-M., Högele, A., Simmel, F. C., Govorov, A. O., Liedl, T. "DNA-based self-assembly of chiral plasmonic nanostructures with tailored optical response". Nature, 483, 311-314, 2012.

[5] Novotny, L., van Hulst, N. “Antennas for light”. Nat. Phot, 5, 83-90, 2011.

[6] Taminiau, T. H., Stefani, F. D., van Hulst, N. F. "Enhanced directional excitation and emission of single emitters by a nano-optical Yagi-Uda antenna”. Opt. Exp, 16, 10858-10866, 2008.

[7] Balanis, C. A., Antenna Theory: Analysis and Design, $3^{\text {rd }}$ Edition. Wiley-Interscience, 2005. 